\title{
Ridge augmentation using mandibular tori
}

\author{
D. Barker, ' A. W. G. Walls, ${ }^{2}$ and J. G. Meechan, ${ }^{3}$
}

\begin{abstract}
A 19-year-old female was referred by her dental practitioner for the restoration of missing maxillary lateral incisors and canines. Ridge augmentation was required. This was undertaken using mandibular tori as the sites for harvesting bone. The grafting was successful and the spaces were subsequently restored using resin-bonded bridgework. The case reports that mandibular tori provide a local and convenient source of bone for ridge augmentation procedures.
\end{abstract}

$\mathrm{W}$ hen planning the replacement of lost or congenitally absent teeth by conventional or implant retained prostheses, consideration must be given to the alveolar ridge form in the edentulous area. This is important in terms of aesthetic considerations and to provide sufficient bone volume for the placement of endosseous implants when indicated. In the anterior maxilla, labial ridge deficiency after tooth loss is almost inevitable and the ridge architecture may need to be re-established if a satisfactory result is to be created.

A number of different methods have been reported to enhance the morphology of the

${ }^{1}$ Specialist Registrar, ${ }^{2}$ Honorary Consultant, Departments of Restorative Dentistry, ${ }^{3}$ Senior Lecturer/Honorary Consultant, Oral and Maxillofacial Surgery, Newcastle Dental Hospital

${ }^{*}$ Correspondence to: Dean Barker, Specialist Registrar in Restorative Dentistry, Department of Restorative Dentistry, Dental Hospital, Richardson

Road, Newcastle upon Tyne NE2 4AZ

email:dean.barker@ncl.ac.uk

REFEREED PAPER

Received 05.06.00; Accepted 06.10.00

(C) British Dental Journal 2001; 190: 474-476 defect and augment the alveolar bone. ${ }^{1-3}$ It is generally felt that bone grafting, replacing 'like with like' is preferable to other techniques such as connective tissue grafting. These can be summarised as: autogenous bone grafts; guided bone regeneration; alloplast graft materials; allografts; xenografts; bone promoting molecules. It is beyond the scope of this report to discuss the relative merits and disadvantages of each of these. However, it is widely considered that autogenous bone grafting techniques are the most predictable and can be thought of as the gold standard against which the other methods are measured. ${ }^{2}$ Autogenous bone has a number of advantages over the alternatives in that it is: readily available from local or distant sites; sterile; biocompatible/ non-immunogenic; osseoinductive/conductive; easy to manipulate. ${ }^{1}$ Extra-oral sites used for harvesting bone include the ileum, tibia, scapula, clavicle and calvarium. Intraorally the chin, mandibular ramus, retromolar regions and other edentulous areas distant to the operating site have been used. This case report describes the use of
In brief

- Labial ridge deficiency in the maxilla is almost inevitable after tooth loss

- Ridge augmentation may be required to produce a satisfactory aesthetic result when bridgework and implant retained restorations are used.

- Augmentation may be essential prior to implant placement in order to produce an adequate volume of bone.

- Mandibular tori may be used for ridge augmentation.

mandibular tori as a source of bone for grafting.

\section{Case report}

A 19-year-old female was referred to the Department of Restorative Dentistry by her general dental practitioner for the restoration of missing maxillary lateral incisors and canines. The canines were impacted and had been surgically removed, at another institution, 4 years previously. Unfortunately during surgery it was also felt necessary to remove the upper lateral incisors bilaterally.

Medically, the patient had an allergy to penicillin, otherwise she was found to have no relevant medical history.

Clinical examination revealed little of note extra-orally. Intra-orally, the mucosa was normal. Bilateral mandibular tori were present (Fig. 1). Oral hygiene level and periodontal health were good. Early occlusal caries was noted in two molar teeth. Radi-
Fig. I

(a) Left mandibular torus, (b) Right mandibular torus
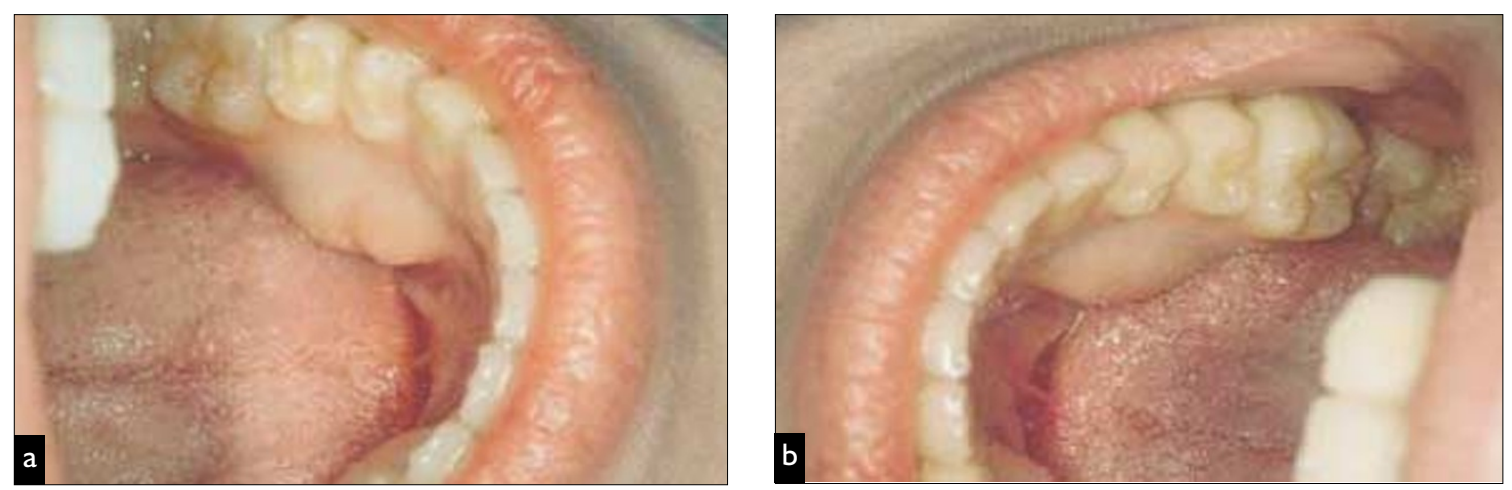


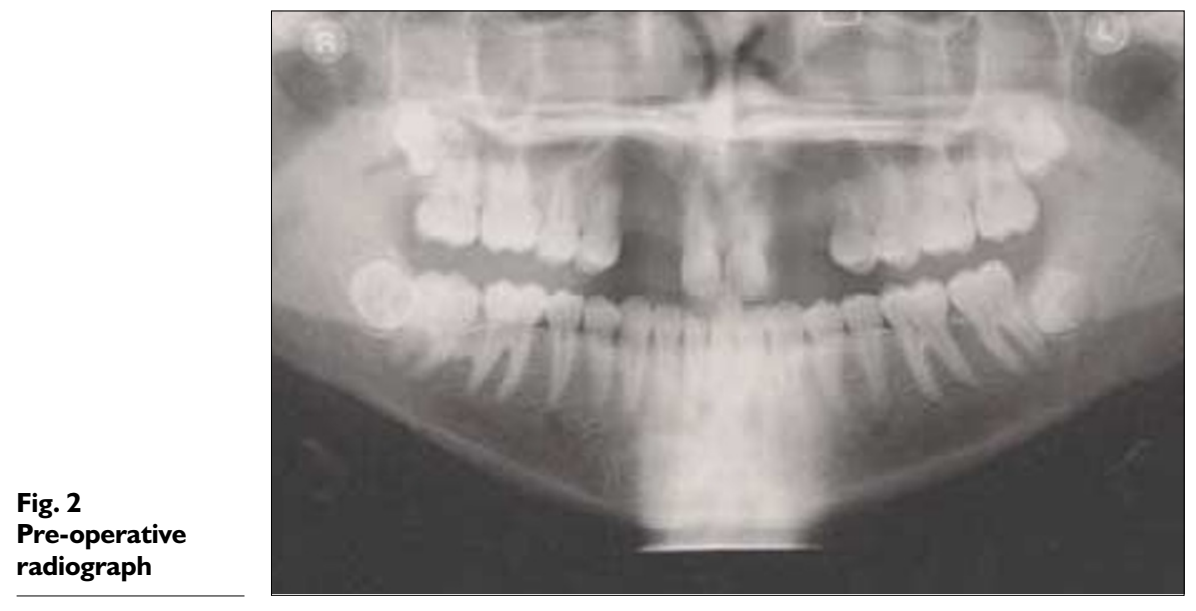

Fig. 3

Pre-operative view showing alveolar deficiencies

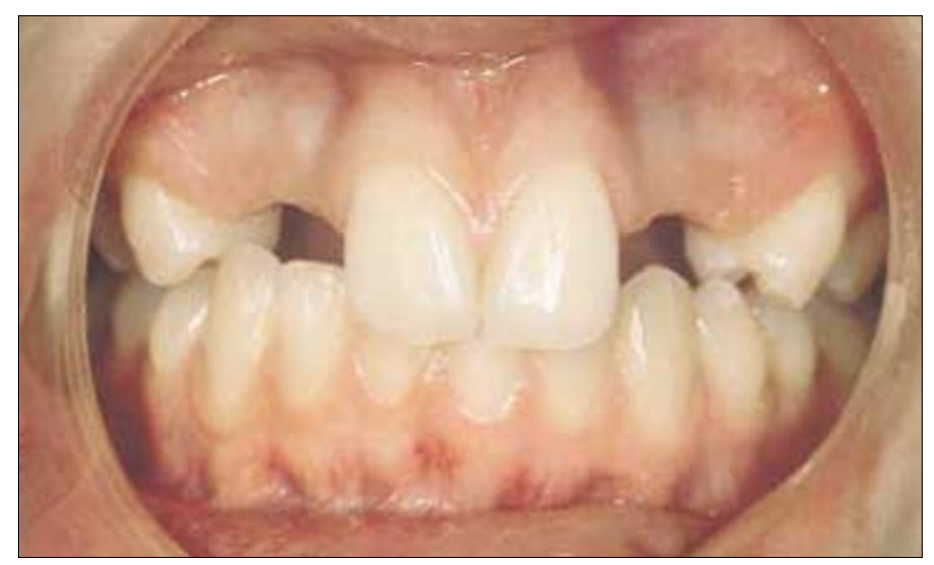

ographic examination revealed that all four third molars were impacted and completely enclosed in bone (Fig. 2). All the remaining dentition was present and unrestored with the exception of the missing maxillary canines and lateral incisors. An upper acrylic partial denture with Adams crib type retention was in place. This prosthesis had a poor appearance. The patient had a high smile line exposing much of the labial gingiva and a clear difference could be detected between the acrylic flanges of the denture and the natural tissues.

The various treatment options were considered. The patient's preferred choice of treatment was simple bridgework rather than endosseous implants. It was decided to replace the missing teeth with resin bonded restorations. There was some deficiency in the width of alveolar bone in the edentulous areas and it was felt that to obtain a satisfactory aesthetic result, alveolar ridge augmen- tation would be necessary (Fig. 3). Without ridge augmentation, the bridge pontics would have to have had either very bulbous or over retroclined necks resulting in an unnatural emergence profile and poor appearance. This was clearly apparent by clinical observation and by consideration of the existing prosthesis. The mandibular tori
Fig. 4 I-week postsurgery. A fixation screw is visible through the mucosa UR3(13) region were identified as the sites from which to harvest bone.

\section{Treatment}

The patient was admitted to hospital for the procedure to be carried out under general anaesthesia. Full thickness mucoperiosteal flaps were raised in the maxilla to reveal the edentulous areas and on the lingual aspect of the mandible bilaterally to expose the tori. The tori were removed by making a groove adjacent to the lingual cortex with a fissure bur followed by sectioning with an osteotome. The donor sites were smoothed to remove any sharp prominences of bone followed by copious irrigation with sterile saline.

The harvested bone required only slight trimming of its shape in order to fit the dimensions of the recipient site. The grafts were fixed in place by the use of a single titanium screw bilaterally.

Primary closure of the soft tissues was carried out at both donor and recipient sites. No post-operative complications arose and at a review appointment one week later healing was seen to be progressing well (Fig. 4). The fixation screws were removed six months post-operatively under local anaesthesia.

The graft produced a very good ridge profile and the edentulous spaces were restored with fixed-fixed adhesive bridgework 10 months after surgery (Fig. 5). At this time no significant resorption of the augmented areas was apparent. The patient was satisfied not only with the finished prostheses and overall appearance, but also with the loss of the tori.

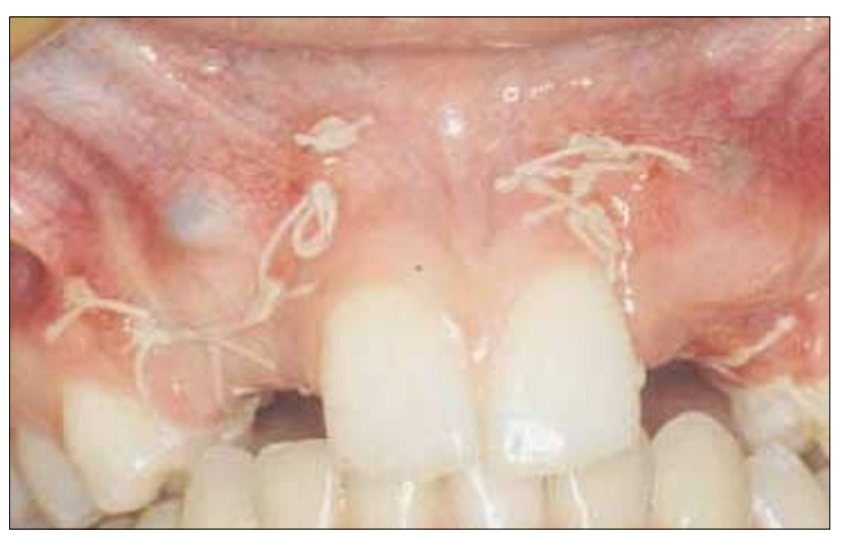




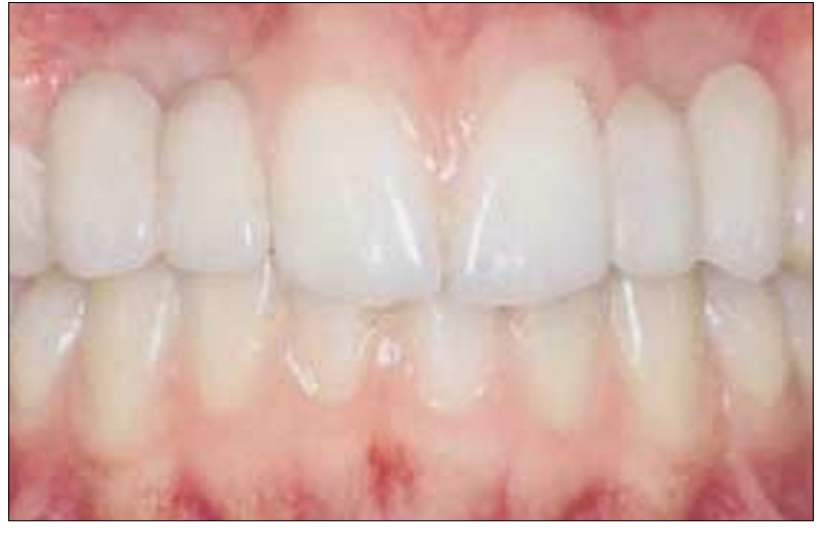

Unfortunately, the bridgework has been subject to a number of failures. In accordance with current clinical practice, the two fixed-fixed prostheses are to be replaced with four adhesive cantilever bridges.

\section{Discussion}

The term exostosis is used clinically to describe a variety of bony outgrowths. ${ }^{4} \mathrm{~A}$ torus is an exostosis that occurs at a characteristic site, either in the midline of the palate (torus palatinus) or on the lingual surface of the mandible (torus mandibularis). It is a non-neoplastic lesion.

Mandibular tori occur in $6-7 \%$ of the population ${ }^{5}$ although there is a variation in prevalence according to race with reports of over $60 \%$ in Eskimos. ${ }^{6}$ They occur with equal frequency between the sexes. Mandibular tori tend to present bilaterally, typically in the premolar region above the mylohyoid line. Their size varies and may extend distally to the third molar and mesially to the lateral incisor region. They may be single, multiple or lobulated and may be composed entirely of cortical bone or be formed by cancellous bone with an overlying shell of cortical bone.

Tori are generally considered of unknown aetiology ${ }^{7}$ although there is evidence to suggest a hereditary component ${ }^{8}$ and their presence has been associated with parafunctional activity. ${ }^{9-11}$ The time required for the development of the lesions in relation to parafunctional habits and the mechanism of development are unknown. In this case, the patient did not experience temporomandibular joint symptoms or migraine and no evidence of these problems were detected on clinical examination.

Pynn et al. ${ }^{12}$ suggested five indications for tori removal: traumatic ulcers from mastication; prosthodontic considerations; cancer phobia; interference with tongue function during mastication; interference with normal speech. The authors suggest that 'prosthodontic considerations' should include the harvesting of the tori for augmentation procedures. In this case the grafting was required for aesthetic reasons alone, but if the case had been treated using the placement of implants, grafting would have been essential to produce an adequate volume of bone. Exostoses are an ideal site for harvesting as the surgery results in no anatomical or aesthetic deficit. Ganz has previously reported the successful use of mandibular tori for onlay bone grafting on the buccal aspect of the mandible, prior to implant placement. ${ }^{6}$

The long-term stability of the grafts is uncertain. Autogenous bone grafts, although the 'gold standard', tend to undergo remodelling and resorption. Grafts that are not loaded for prolonged periods, as in this case, may experience unnecessary resorption because of lack of functional stimulation. Even when implants are placed, resorption will occur. With implants, most clinicians recommend progressive loading to minimise the resorption. However, no resorption of the grafted bone has been seen to have taken place in the first 2 years following surgery.

Possible complications of surgery include lingual nerve damage in cases of distally extended tori, infection and floor of mouth haemorrhage. The latter is a rare sequela but can be life threatening and must be managed immediately in order to prevent airway embarrassment. $^{13}$

\section{Conclusion}

Mandibular tori provide a local and convenient source of bone for ridge augmentation procedures.

1 Palmer P, Palmer R. Implant surgery to overcome anatomical difficulties. Br Dent $J$ 1999; 187: 532-540.

2 Rissolo A R, Bennett J. Bone grafting and its essential role in implant dentistry. Dent Clin North Am 1998; 42: 91-116.

3 Buser D, Dulak K, Hen D, Hirt H P, Belser U C. Localised ridge augmentation with autografts and barrier membranes. Periodontol 2000 1999; 19: 151-163.

4 Soames J V, Southam J C. Oral pathology. 2nd ed. p313. Oxford: Oxford University Press, 1993.

5. Kolas S, Halperin V, Jeffries K R et al. Occurrence of torus palatinus and torus mandibularis in 2,478 dental patients. J Oral Surg 1953; 6: 1134.

6 Ganz S D. Mandibular tori as a source for onlay bone graft augmentation: a surgical procedure. Practical Periodontics Aesthetic Dent 1997; 9: 973-982.

7 Hrdlicka A. Mandibular and maxillary hyperostoses. Am J Phys Anthropol 1940; $27: 67$.

8 Eggen S. Torus mandibularis: an estimation of the degree of genetic determination. Acta Odontol Scand 1989; 47: 409-419.

9 Eggen S. Torus mandibularis og muskulaer hyperaktivitet. Nor Tannlaegeforen Tid 1988; 98: 220-226.

10 Ossenberg N S. Mandibular torus: a synthesis of new and previously recorded data and a discussion of its cause. In Cybulski J S (ed) Contribution to Physical anthropology 1978-80 pp1-52. Ottowa: National Museum of Canada, 1981.

11 Clifford T, Lamey P-J, Fartash L. Mandibular tori, migraine and temporomandibular disorders. Br Dent J 1996; 180: 382-384.

12 Pynn B R, Kurys-Kos N S, Walker D A, Mayhall J T. Tori mandibularis: a case report and review of the literature. J Canad Dent A 1995; 61: 1057-1066.

13 Hull M. Life-threatening swelling after mandibular vestibuloplasty. J Oral Maxillofac Surg 1970; 28: 565. 